\title{
An overview on bone protein extract as the new generation of demineralized bone matrix
}

\author{
ZHOU ZhiYu ${ }^{1}$, ZOU LiJin $^{2}$, LI HaiSheng ${ }^{3}$, BÜNGER Cody $^{3} \&$ ZOU XueNong $^{1 *}$ \\ ${ }^{1}$ Orthopaedic Research Institute/Department of Orthpaedic Surgery, First Affiliated Hospital of Sun Yat-sen University, Guangzhou 510080, \\ China; \\ ${ }^{2}$ Department of Burn Surgery, First Affiliated Hospital of Nanchang University, Nanchang 330031, China; \\ ${ }^{3}$ Orthopaedic Research Laboratory/Department of Orthpaedic Surgery, Aarhus University Hospital, Aarhus 8000, Denmark
}

Received May 27, 2011; accepted November 15, 2012

\begin{abstract}
Bone protein extract is regarded as the new generation of demineralized bone matrix. The aim of this paper is to describe and characterize the properties of demineralized bone matrix and its new generation product in addition to its application in animal and human studies. Bone protein extract has features of osteoconductivity, osteoinductivity and osteogenicity, which originate from its unique and precise processing. It has exhibited powerful bone formation capacity both in animal experiments and in clinical trials by providing an optimal microenvironment for osteogenesis. Furthermore, not only does it have excellent biocompatibility, it also has good compatibility with other implant materials, helping it bridge the host and implanted materials. Bone protein extract could be a promising alternative for demineralized bone matrix as a bone graft substitute.
\end{abstract}

bone protein extract, demineralized bone matrix, autologous bone graft, osteogenesis

Citation: Zhou Z Y, Zou L J, Li H S, et al. An overview on bone protein extract as the new generation of demineralized bone matrix. Sci China Life Sci, 2012, 55: 1045-1056, doi: 10.1007/s11427-012-4415-7

With the development of modern medical techniques, most orthopedic diseases can be treated effectively. However, most failed orthopedic operations result in the non-union of bone. Although autologous bone grafting can provide good results and has been being regarded as the gold standard [1-5], complications associated with bone graft harvesting, such as neurovascular injury [6], deep wound infection, hematoma, peritoneal perforation, ureteral injury $[7,8]$ and chronic pain at the donor site [9-15], are still major concerns $[10,16]$. There are many bone graft substitutes available, including coral hydroxyapatite, calcium phosphate, calcium sulfate and nano-hydroxyapatite collagen; however, none of these possess all three features required of an optimal bone biomaterial, which are osteoinductivity, osteoconductivity and osteogenicity.

*Corresponding author (email: zxnong@ hotmail.com)
Demineralized bone matrix (DBM) is one of the few bone alternatives that exhibit osteoinductive, osteoconductive and osteogenicity characteristics [17-24]. When incorporated with internal fixation devices and bone marrow mesenchymal stem cells, it can provide the ideal microenvironment for bone formation. In the late 19th century, Senn [25] was the first to report that demineralized bovine xenografts could be successfully used to repair long bone and cranial defects in dogs and femoral and tibial defects in humans [20]. The osteoinductive properties of DMB were not confirmed until a 1965 study by Urist et al. [26]. A 2009 study by Katz et al. further increased understanding of bone morphogenetic proteins (BMPs) [18].

Although DBM is deemed effective enough to be used in clinical applications, there are a number of limitations preventing its widespread clinical use. First, there is a limit to the amount of allograft bone available to produce DBM [27] 
and second, the production of DBM from other species raises ethical concerns. Fortunately, DBM is not species-specific and from this bone protein extract (BPE), the new generation of DBM, has been removed. In 2007, the first commercial BPE was certified by the Food and Drug Administration (FDA) in the United States. This can be combined with a PEEK interbody cage for clinical use in Europe. In this review, we take an in-depth insight into the biological properties of BPE and their use in orthopedic surgery for further understanding of DBM and the new generation product.

\section{Biological characteristics of bone protein ex- tract}

Bone protein extract is a new form of biomaterial extracted from DBM isolated from the extracellular matrix of the compact tissue of long bone [28]. This biomaterial is a lyophilized form of collagenous matrix, consisting of collagen type I chains and other insoluble proteins, including transforming growth factor-beta (TGF- $\beta$ ) superfamily, among other factors. It has a cotton-like appearance, with a white to slightly yellowish color, is processed under aseptic conditions and is non-pyrogenic.

\subsection{The extraction of BPE}

BPE is created from the extracellular matrix of animal long bones (i.e., humerus, radius, metacarpus, femur, tibia, fibula and metatarsus). Although the first commercial BPE was made from equine bone, the first clinical BPE was derived from bovine bone. Because of concerns regarding bovine spongiform encephalopathy, it is very difficult to pursue the clinical application of BPE from bovine bone [29].
BPE is aseptically produced by a series of precise and complicated processes. The health of donor animals and their exposure to pathogens is continuously monitored and documented throughout [30]. Cortical diaphyses of long bones are used to create BPE following the removal of the soft tissue and marrow [29]. Clean bone segments are pulverized and delipidated with acetone three times for 60 minutes each at $2-7^{\circ} \mathrm{C}$. Then the pulverized bone is demineralized in $0.6 \mathrm{~N}$ hydrochloric acid three times for 60 minutes each at $3-10^{\circ} \mathrm{C}$. Following demineralization, the particles are washed in triple-distilled water and the extracted proteins are isolated from a $4 \mathrm{~mol} \mathrm{~L}^{-1}$ guanidine hydrochloride solution buffered with trishydroxymethylaminomethane (TRIS), ethylenediaminetetraacetic acid (EDTA), and hydrogen chloride solution ( $\mathrm{HCl}), \mathrm{pH} 6.9$, at $15-20^{\circ} \mathrm{C}$ for $12.5 \mathrm{~h}$ for two repetitions and once for $250 \mathrm{~min}$. After purification using a 3000 nominal molecular weight cut-off ultra filter, the extracted proteins are lyophilized, resulting in a cotton-like appearance [31,32] (Figure 1).

Compared with the production of traditional DBM, the production of BPE has the following characteristics which are beneficial for its use: (i) The bone used is sourced from documented animals; (ii) the production process is precisely designed and rigidly observed; (iii) unlike the traditional single demineralization step, there are two dematerialization steps in the processing of BPE, using both $\mathrm{HCl}$ and EDTA $[26,33,34]$; (iv) the extracted proteins are lyophilized in the final step. Owing to these specialized processing steps, BPE possesses unique features making it a more ideal bone graft substitute than traditional DBM.

\subsection{Composition analysis of bone protein extract}

Although DBM was first used in the late 19th century and later shown to be effective for bone fusion in 1965, its use

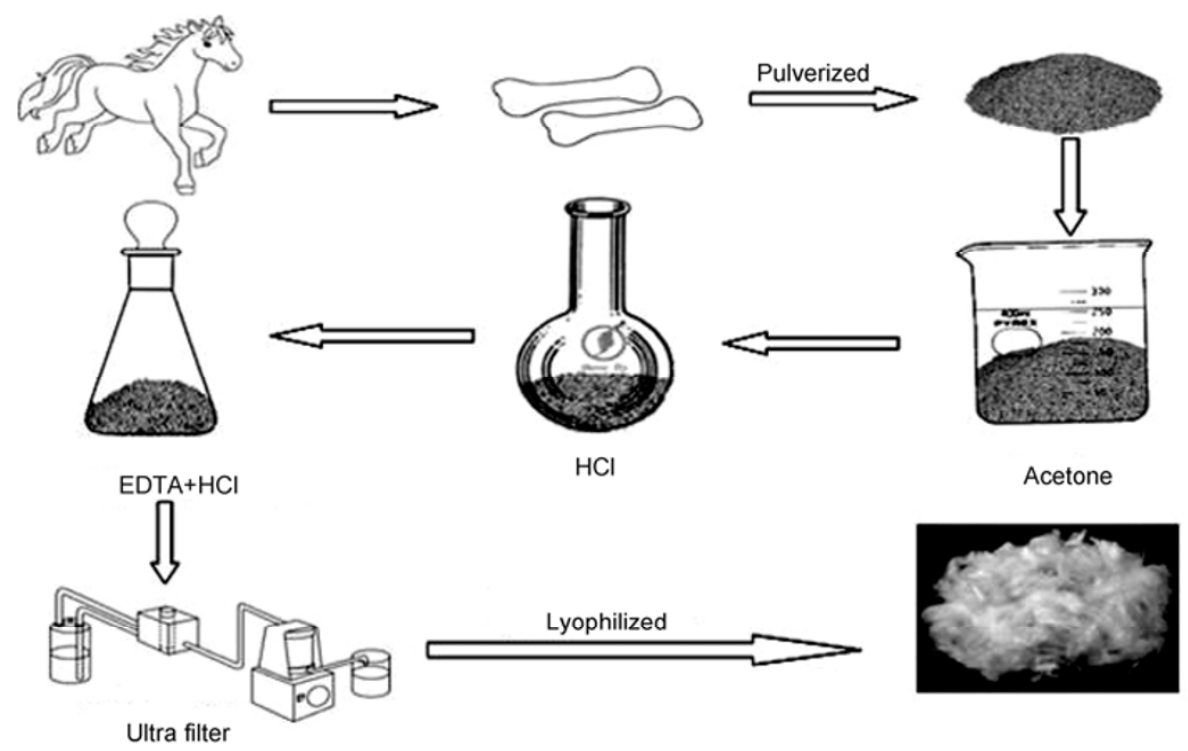

Figure 1 Extraction process of bone protein extract. $\mathrm{HCl}$, hydrochloric acid; EDTA, ethylenediaminetetraacetic acid. 
in promoting bone formation was not fully examined until BMPs were discovered by Urist in 1975. Wildemann et al. [35] demonstrated that DBM, which has been demineralized and sterilized by different processing methods, retained different amounts of insoluble proteins by comparing three commercially available DBMs. However, in those studies, BMP-2 was detected at its highest level in all DBMs tested. Honsawek et al. [36] measured and quantified BMP-4 in DBM from human bone, and they demonstrated that the osteoinductive capacity of DBM increased corresponding to BMP-4 concentration by heterotopic ossification in athymic mice. Bae et al. [37] compared BMPs in several commercial DBMs and found that BMP-2 and BMP-7 could be detected in all products at different concentrations. The different extraction methods used in the production of each DBM were considered to be the most important factor in determining the BMP content of DBM. William et al. [21] also measured BMP-4 in DBM, but found it was lower than BMP-2 and BMP-7. All three BMPs were confirmed by mass spectrometry or ELISA in BPE, but were not detected by western blotting by Nienhuijs et al. [32]. In their study, BMP-2 was approximately one hundred-fold higher in BPE than in DBM. Thus, to date, at least three BMPs have been verified to be present in BPE. With the development of this technique, more BMPs may be detected in BPE by high-tech precision instruments in the near future.

Regarding bone formation, BMPs, TGF- $\beta$, a member of the TGF superfamily, also plays an important role in bone regeneration. Several TGF- $\beta$ s have been detected in both DBM and BPE. Carrington et al. [38] demonstrated that TGF- $\beta$ s were present in DBM following extraction with EDTA-guanidine hydrochloride, but not guanidine hydrochloride alone. Wildemann et al. [35] verified that TGF- $\beta 1$ was present in all the three commercial DBMs, although it was at a low level. Katz et al. [18] found a weak Spearman correlation between the level of TGF- $\beta 1$ in the DBM of donors' bone and its capacity for bone formation. However, we believe that multivariate correlation analysis should be used as TGF- $\beta 1$ was just one of the microenvironmental factors contributing to enhanced bone formation. Using mass spectrometry and western blotting simultaneously, Nienhuijs et al. [32] found that TGF- $\beta 1$ and TGF- $\beta 2$ were all present in BPE, with TGF- $\beta 1$ being over two hundred-fold higher than in DBM.

TGF- $\beta 1$ and TGF- $\beta 2$ are the main insoluble proteins contributing to the osteoinductive capacities of BPE. Indeed, there are some known and undetected trace proteins present in BPE, such as insulin-like growth factor 1(IGF-1), which also play a role in creating a suitable microenvironment for bone formation. However, these factors are not functional unless they are present in osteoconductive media, mainly type I collagen, where they can exert their functions. In the early 1970s, Bachra [39] verified that bone collagen was a good nucleation catalyst for mineral deposition compared with rat tail collagen. Hanson et al. [40] studied the stereo- specific features of type I collagen in human bone, which is convenient for collagen mineralization. Although there has been no scientific paper on type I collagen in BPE, it has been documented by the FDA.

Thus, BPE is composed of a collagenous matrix, including type I collagen chains and several other insoluble proteins, such as BMP-2, BMP-3, BMP-7, TGF $\beta-1$, TGF $\beta-2$, IGF-1 and other unknown factors. These components endow BPE with all the bone formation activities, as it displays good osteogenicity, osteoconductivity and osteoinductivity. Compared with other mono-factors such as BMP-2, BPE has the following unique advantages: (i) Animal bone is abundant, and the health and exposure to pathogens of donor animals is continuously monitored and documented, which enable the safe and effective mass production of BPE; (ii) most mono-factors are only effective in very high concentrations, with several authors having reported on heterotopic ossification induced by BMPs [41-43]. BPE has not be shown to have this effect, although the dose-effect relationship of BPE is difficult to define in different patients; (iii) besides autologous bone grafting, DMB and this new generation product are the only products that can provide optimal microenvironments for bone formation.

\subsection{Bioactivity}

The ideal bone graft material must possess three key properties; osteoconductivity; osteoinductivity: and osteogenicity [44]. Autologous bone grafting is the only procedure that currently meets all three of these requirements. However, BPE shows good characteristics of all the three of these properties, which may be increased by coupling with stem cells for more powerful osteoinductive and osteoconductive capacities, which is far beyond other DBMs.

The osteoinductivity of a graft material is its ability to facilitate the recruitment and differentiation of stem cells into osteoblasts (the bone-forming cells) [44]. Although stem cell recruitment has not been extensively examined in orthopedics, Urist [26] demonstrated that three distinct populations of cells were recruited in the host tissue and among these were osteoprogenitor cells, which are regarded as stem cells. Using BPE in the anterior spinal fusion cage, $\mathrm{Li}$ et al. [45] found that it produced the same effects as autologous bone grafting in achieving fusion and the percentage of new bone formation. The excellent effectiveness of BPE in this study was believed to result from the migration and differentiation of mesenchymal cells from the vertebral bodies [45]. This hypothesis was confirmed in similar experiments by Walboomers et al. [46]. A further study demonstrated that the mechanisms of bone formation with BPE were related to increased penetration of blood vessels into these materials than that into other bone substitutes, leading to a rapid enrichment of osteoblasts and an induction of membranous bone formation [47]. In combination 
with DBM from human bone, Kuvat et al. [48] used BPE as an osteoinductive material in clinical maxillofacial surgery to good results, which were evaluated by clinical and histopathological examination.

Osteoconductivity is the ability of graft materials to provide biocompatible scaffolding that supports new bone formation and subsequent growth [44]. The interaction between cells and bone substitutes is reliant on the attachment, adhesion and spreading of bone cells on the material, and the ability to further influence cell proliferation and differentiation. Type I collagen, which contains Arg-Gly-Asp (RGD) sequences, is the ideal biomaterial component [49-51]. Several studies have verified that collagen can enhance the adhesion and contact guidance of osteoblasts inoculated onto the composite surface through specific RGD sequences that mediate interaction between integrins and the substrate surface thereby promoting cell adhesion [49-51]. Collagen in DBM has also been confirmed to have osteoconductive functions [39]. Hanson et al. [40] found that the stereospecific features of bone collagen at the molecular level, such as the pattern of cross-linking and the interchain placement of bonds, were markedly different from other tissue collagens, in particular type I collagen, which may alter collagen mineralization. As BPE is a lyophilisate in collagenous matrix form, consisting of collagen type I chains and other insoluble proteins, the osteoconductive capacity is obvious.

An osteogenic bone graft contains cellular elements at different stages of osteoblastic differentiation, including osteoprogenitor cells, that are capable of synthesizing new bone at the fusion site, thus forming new bone directly $[25,44]$. Currently, none of the bone substitutes available have the osteogenic capacity that autogenous bone grafting has, and BPE is certainly no exception. However, when incorporated with stem cells, DBM has increased bone forming abilities, as the stem cells impart a semi-osteogenic capacity to it. Becerra et al. [52] demonstrated that bone marrow stromal cells are more osteogenic in the presence of DBM, whereas they tended to form other colonies, such as fibroblastic, epithelial or smooth muscle cells, without it. The combined osteogenicity of DBM and stem cells was further studied by many authors. In their experiments, the extent of bone formation induced by DBM combined with stem cells was obviously increased when compared with that induced by stem cells or DBM alone [53-60]. BPE could increase the proliferation of undifferentiated stem cells and accelerate the differentiation of pre-mature osteoblastic cells in vitro [61]. To the best of our knowledge, no report has been published on the use of combined stem cells and BPE for treatment in vivo.

\subsection{Stem cell interaction}

DBM, and in particular its new generation product BPE, has been shown to promote the proliferation and differentiation of stem cells. Kasten et al. [62] seeded bone marrow stromal cells (MSCs) on calcium-deficient hydroxyapatite (CDHA), $\beta$-tricalcium phosphate ( $\beta$-TCP) and DBM. DBM was an excellent matrix for MSCs according to the cells seeding efficacy or osteocalcin expression, compared with synthetic materials. These findings were supported by a number of other studies [62-65]. By culturing human mesenchymal stem cells over-expressing human telomerase reverse transcriptase (hMSC-TERT) in plates coated with BPE at different concentrations, Woo et al. [61] demonstrated that BPE could increase the proliferation of undifferentiated hMSC-TERT cells and accelerate osteoblastic differentiation of hMSC-TERT cells with a limited effect on proliferation. It was also observed by El-Sabban et al. [66] that BPE could induce osteoblastic differentiation of in vitro expanded BMSCs.

Accordingly, the osteoinductive and semi-osteogenic capacities enable BPE to induce the proliferation and differentiation of stem cells, thus promoting bone formation. However, these effects are not just seen on stem cells but on other cells, such as L-929 fibroblasts and the muscle-derived cell line $\mathrm{C} 2 \mathrm{C} 12[67,68]$. One point worth noting is that unlike other DBM, the interaction between BPE and stem cells, to the best of our knowledge, has not been shown in vivo, and thus further studies are warranted.

\subsection{Combination of other materials}

BPE has a poor ability to retain its shape in vivo without providing mechanical support. Thus, a carrier is needed to help define the shape of the implant, while also retaining the proteins in the specific area for an adequate time period [69]. Owing to this, it is not worth applying BPE alone for large bone defects even though it is much cheaper than rhBMPs. In addition, using too high a concentration could theoretically result in heterotopic ossification, which can occur with BMPs [41-43,70]. More importantly, as BPE is made from a natural organism, it has been verified to have good biocompatibility with host tissue.

Furthermore, BPE has good synergistic actions with other materials. A canine study demonstrated that fibrous tissue covered $\beta$-TCP implants, which hindered implant degradation and new bone formation. $\beta$-TCP in combination with $\mathrm{BPE}$ was protected from this phenomenon and gave a two-fold increase in mechanical implant fixation [71]. Some authors observed a synergistic effect between BPE and carboxymethylcellulose (CMC), where they reported that a $3 \%$ CMC gel could act as carrier or bulking agent either in gel or lyophilized form $[28,69]$. However, we should not expect the best results for the use of BPE in either of those conditions. In vitro, the combination of BPE with a collagen hydroxyapatite composite scaffold resulted in a synthetic bone graft substitute, which could be completely remodeled into vital bone tissue [72]. However, the same carrier, which was derived from an equine source, unexpectedly inhibited the 
osteoinductive capacity of BPE in vivo as a result of an inflammatory response [73]. To the best of our knowledge, this is the first and only report to date where BPE had a negative effect of bone formation.

Although several authors have studied different carriers for BPE, more research needs to be performed to determine the best carrier for orthopedic clinical applications. Further investigations should focus on determining the most suitable carrier, both in basic research and multicenter clinical studies.

\subsection{Molecular mechanism of bone formation}

Bone formation is a complex, sequential and overlapping process. Although many factors have been verified to accelerate bone formation, the mechanism and their interactions are still poorly understood. Zou et al. [74] pioneered this field by using microarray technology to examine gene-expression profiles during anterior lumbar interbody fusion (ALIF) after implantation of different graft materials, including BPE, recombinant human bone morphogenetic protein-2 (rhBMP-2) and autograft bone. They found that different pathways induced different kinds of bone formation at different time points and in different situations. Compared with autograft bone, rhBMP-2 induced membranous ossification via recruitment, proliferation and differentiation of osteoblastic progenitor cells by upregulating various factors, such as prostaglandin endoperoxide $\mathrm{H}$ synthase-2 (PGHS-2), insulin-like growth factor binding protein-2 (IGFBP-2), vascular endothelial growth factor (VEGF) and chemokines. In contrast, BPE resulted in endochondral ossification via the upregulation of cartilage- related genes, which is similar to autograft bone.

Therefore, BPE, distinct from other artificial bone graft materials, can provide the most optimal biological microenvironment for bone formation. With the technology available, it is currently not possible to build a biological microenvironment without using natural bone because of the complexity of bone. Some factors, such as rhBMP-2, may improve bone formation, but it does not reform in a natural manner. Thus, as BPE originates from natural bone, it may be a better choice.

\subsection{Biocompatibility}

Bone is known to possess low immunogenicity. Thus, bone allograft transplantation has been quite successful for several decades, with cryopreserved bone in particular being quite effective [75-77]. Even today, this treatment remains necessary under certain circumstances. BPE also possesses the characteristic of having lower immunogenicity. BPE was shown to have good cytocompatibility, in combination with human mesenchymal stem cells, as cells could adhere, proliferate and differentiate into the osteoblastic lineage [72]. Although BPE is made from bovine or equine sources, it still has excellent biocompatibility when transplanted into different species, including humans (Table 1). Furthermore, BPE is compatible with titanium implants, which can bridge the inorganic material and local tissue [45,46,78-80]. Therefore, the good biocompatibility of BPE can not only accelerate bone formation, but also enhance the stability of the implants by being compatible with both the bone tissue and the implant.

Table 1 Bone protein extract used in different animal experiments

\begin{tabular}{|c|c|c|c|c|}
\hline Author & Journal & $\operatorname{Animal}(n)$ & Observation time & Comments \\
\hline Baas et al. [101] & J Biomed Mater Res A & $\operatorname{Dog}(9)$ & 4 weeks & No complications \\
\hline Ding et al. [102] & $\begin{array}{l}\text { J Biomed Mater Res B } \\
\text { Appl Biomater }\end{array}$ & Sheep $(8)$ & 9 weeks & $\begin{array}{l}\text { Complications of a paralytic hind leg in one sheep, no related to } \\
\text { BPE }\end{array}$ \\
\hline Jensen et al. [28] & Open Orthop J & Sheep $(6)$ & 16 weeks & No complications \\
\hline Nienhuijs et al. [29] & $\begin{array}{l}\text { J Biomed Mater Res B } \\
\text { Appl Biomaterials }\end{array}$ & $\operatorname{goat}(6)$ & 9 weeks & Swelling for two weeks, the animals ate normally \\
\hline Nienhuijs et al. [73] & Tissue Eng Part A & $\operatorname{rat}(18)$ & 12 weeks & Redness and swelling of the skin but good health \\
\hline Zou et al. [74] & Biomaterials & $\operatorname{Pig}(18)$ & 8 weeks & No comments \\
\hline Baas et al. [71] & Acta Orthop & $\operatorname{Dog}(10)$ & 4 weeks & $\begin{array}{l}\text { One dog was excluded from the study due to a postoperative frac- } \\
\text { ture of the right humerus, the remaining } 9 \text { dogs were without } \\
\text { complications }\end{array}$ \\
\hline Foldager et al. [90] & Spine & $\operatorname{Pig}(18)$ & 8 weeks & One infection \\
\hline Li et al. [89] & $\begin{array}{c}\text { J Biomed Mater Res B } \\
\text { Appl Biomater }\end{array}$ & $\operatorname{Pig}(10)$ & 12 weeks & No comments \\
\hline Baas et al. [79] & Acta Orthop & $\operatorname{Dog}(16)$ & 4 weeks & No postoperative complications \\
\hline Nienhuijs et al. [80] & Biomaterials & $\operatorname{rat}(18)$ & 12 weeks & $\begin{array}{l}\text { One animal died because of anaesthesia, other rats appeared to be } \\
\text { in good health }\end{array}$ \\
\hline Li et al. [45] & Spine & $\operatorname{Pig}(12)$ & 12 weeks & $\begin{array}{l}\text { Two of the pigs were terminated at the fourth week: one resulting } \\
\text { from abdominal wall infection and another resulting from de- } \\
\text { creased activity probably caused by pain }\end{array}$ \\
\hline Walboomers et al. [46] & Biomaterials & $\operatorname{rat}(18)$ & 12 weeks & Good health \\
\hline
\end{tabular}




\section{Animal study}

Many experiments in animals have confirmed the bone formation effects of DBM. To find the best way for bone decalcification, Urist [26] performed experiments in over 200 rabbits and successfully verified these results in several other animal species. Almost all subsequent experiments involving DBM were based on his original findings, including BPE. As the new generation of DBM, BPE has excellent performance in bone formation. Several studies have demonstrated that it has the ability to induce ectopic bone formation in different animal models [46,69,73,80,81].

\subsection{Spinal fusion}

Used in several animal spinal fusion models, traditional DBM has been reported to result in good bone formation [82-85]. However, few studies could confirm that DBM is more or even as effective as autografts and thus it could not be recommended to totally replace autografting [86]. Indeed, BPE also has not been verified to have a stronger bone formation capacity than the 'gold standard' autografting.
However, BPE is different from traditional DBM, and a series of studies focusing on spinal fusion using BPE have shown promising results. The lumbar spine fusion model was first built in pigs (Figure 2), which has a reported fusion rate close to that of humans $[87,88]$. Spinal fusion was evaluated by radiography, micro-CT and histomorphometry, and they concluded that BPE achieved the same fusion rate and the same percentage of new bone formation as that of an autologous iliac bone graft in this demanding porcine anterior spinal fusion model after 12 weeks (Figures 3 and 4) $[45,78,89]$. Following these studies, researchers introduced positron emission tomography/computed tomography (PET/ CT) as a new evaluation method (Figure 5) [90] and experimentally investigated the mechanism by examining osteogenic pattern, gene expression and the pathway involved in new bone formation induced by BPE [74,91].

\subsection{Bone defects}

To date, there has been no research reporting on the treatment of large bone defects with BPE. The main reason behind this may be that it may be too difficult for BPE alone

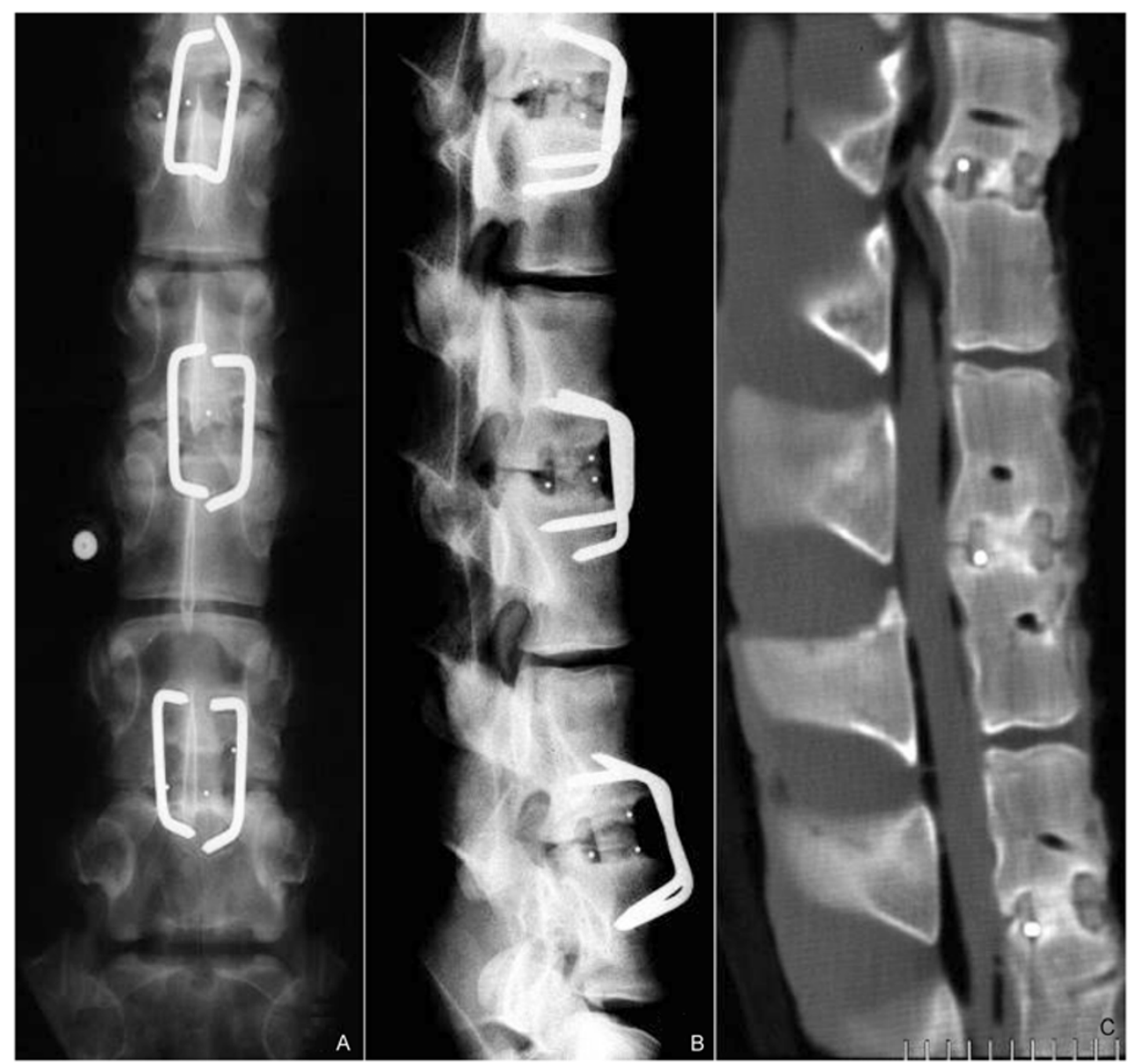

Figure 2 Plain radiographs (A and B) and 3D reconstruction image (C). The cage with bone protein extract and stable construct in a lumbar spine fusion model of pigs. 

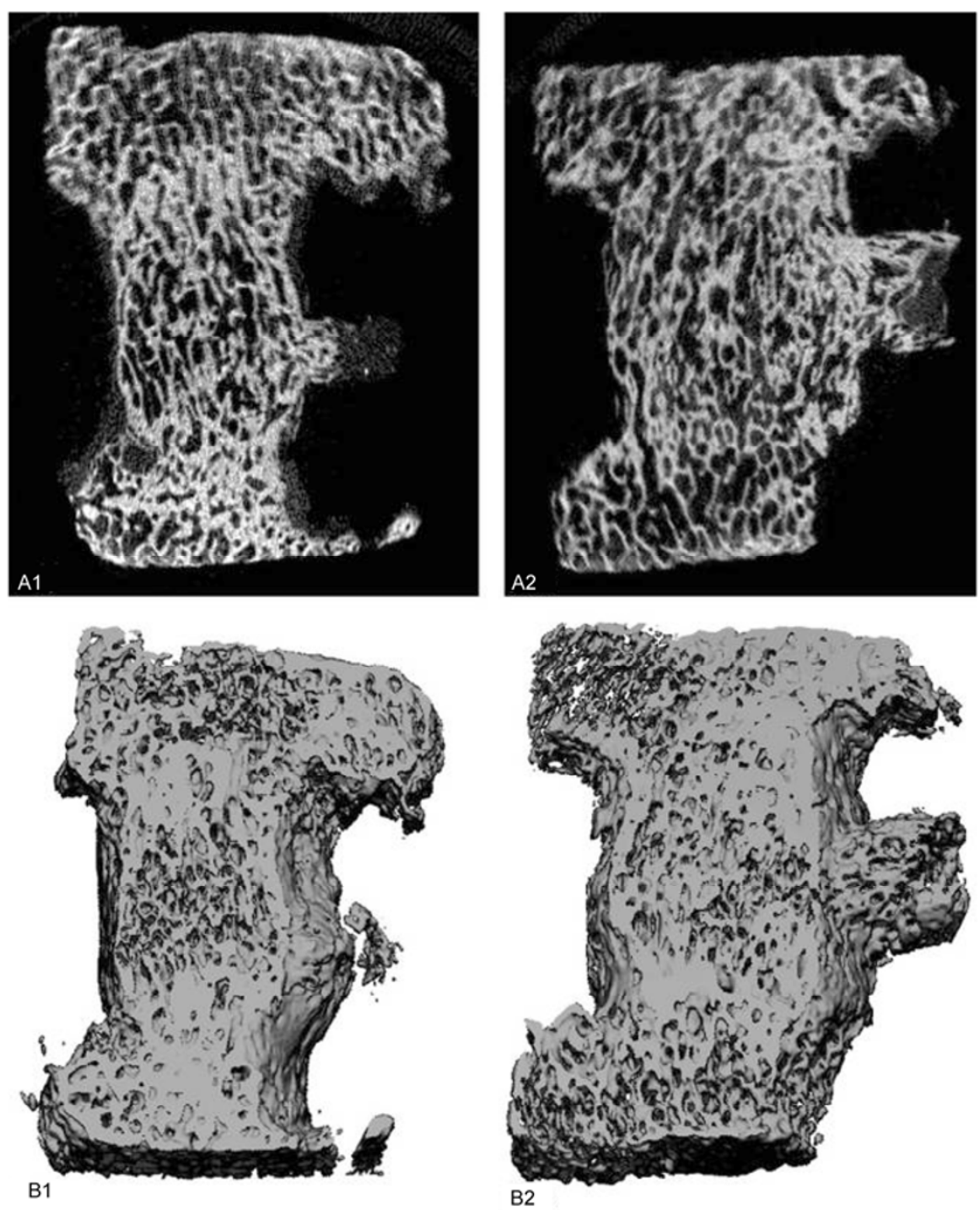

Figure 3 Images of micro-CT of a pig model. The metallic walls of the cage had been cut apart and the bony struts inside the cage were left intact. Both bone protein extract (A1) and autograft (A2) achieved solid fusion with longitudinally organized trabeculae, as evidenced by 2D images (A1 and A2) and 3D reconstructions (B1 and B2). Reproduced from Li et al. [45] with permission from Wolters Kluwer Health.

to deal with an injury of this magnitude without a carrier for structural support. Even so, several authors have reported the effect of BPE in smaller bone defects. Kloss et al. $[92,93]$ applied BPE in treating a porcine forehead defect and the results showed an acceleration of bone regeneration until 26 weeks, which were comparable with autogenous bone grafting. Schlegel [94] observed similar results in his experiments. By placing BPE in 5-mm cortical bone defects in sheep long bone, Huffer et al. [30] confirmed that both bovine and equine BPE was equivalent to autografting, and superior to $\beta$-TCP, in treating a sheep model. Jensen et al. [28] also demonstrated in sheep that BPE had bone regenerative effects in drill defects on the humerus or skull.

Thus, BPE obviously possesses the capacity for treating bone defects. Designing a perfect carrier would be a particular advantage for its use in the treatment of large bone de- fects [72].

\subsection{Artificial joint revision}

In general, autologous bone transplantation is not used in initial joint replacement as there is little bone that can be easily harvested from the operation site. For revision surgery, the situation is entirely different. Artificial joint revision requires huge amounts of bone graft material. Autologous bone is regarded as the best bone graft material; however, it is not easy to obtain enough bone for this kind of operation. Therefore, bone graft substitutes must be used and they should have good biocompatibility with the new prosthesis. Fortunately, Baas et al. [71,79,95] thoroughly studied the biocompatibility of BPE for bone repair. They designed porous-coated titanium alloy implants, which they 

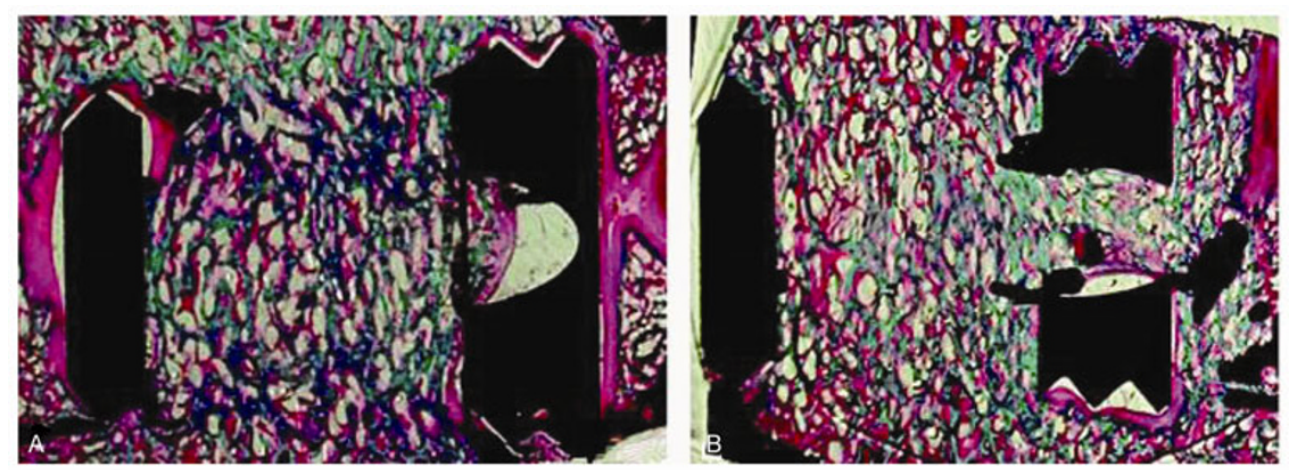

Figure 4 Micrographs from histological sections of pig 12 after three months. Both at the autograft level (A) and bone protein extract level (B), histologic fusion with the trabeculae orientated in the longitudinal direction as a result of mechanical loading (basic fuchsin and light green stain, original magnification $\times 1.25$ ). Reproduced from Li et al. [45] with permission from Wolters Kluwer Health.
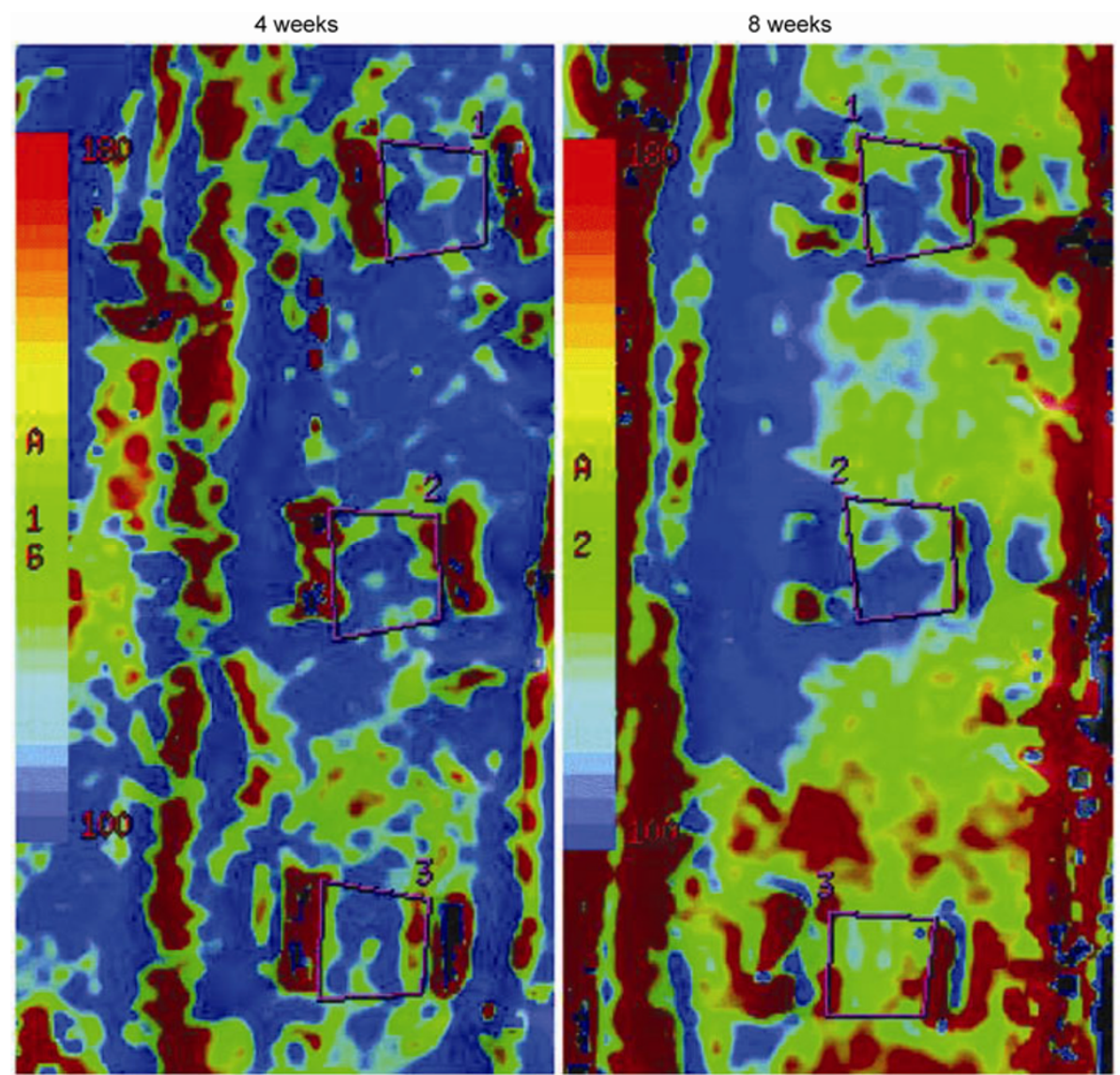

Figure 5 T2 map of a pig model. Regions of interest are marked inside the cages. 1, autograft; 2, bone protein extract; 3, INFUSE. Reproduced from Foldager et al. [90] with permission from Wolters Kluwer Health.

combined with different kinds of bone substitute material, and placed the implants in the lateral proximal humerus of dogs. Using mechanical testing and histological sectioning, authors reported encouraging results showing that BPE could improve mechanical implant fixation, eliminate fibrous tissue formation almost completely, and increase the osseous anchorage and bone remodeling of allografted porous-coated titanium implants. The promising experimental results of the use of BPE offer a new way to augment revision prosthesis.

In summary, BPE induces good bone formation in animal experiments at a number of different surgical sites. All of these studies have provided the basic data for the clinical application of BPE. Aside from the present use of BPE in the clinic, further studies should focus on the following areas: (i) Designing a carrier for BPE and identifying dose- 
effect relationship; (ii) stimulating osteogenic differentiation of stem cells with BPE in vivo; (iii) injecting BPE with an available carrier in the area of the bone defect at different time points; (iv) combining BPE with antibiotics for the treatment of osteomyelitis and, in particular, bone tuberculosis.

\section{Clinical applications}

Although the use of DBM as a bone substitute in clinical applications was first suggested over a century ago, it was not until recently that it received FDA approval. The choice of raw material for DBM has always been a subject of feverish debate. Obviously, there are two sources, animal (xenograft) or human (allograft) bone. In theory, human bone is better but there are a number of limiting factors. First, disease could be transmitted from the donor to the recipient [96]. Second, the limited quantity of human bone prevents its large-scale commercial production. In contrast, animal bone may be a good alternative. Thus, BPE emerged as the solution.

BPE was first reported to be used clinically in spinal fusion in 2002 [97]. When BPE was filled in carbon cages, the fusion rate was $98.6 \%$, higher than that achieved using an iliac crest bone graft. However, there have been no reports on the clinical use of BPE in the treatment of long bone and joint fractures, as a suitable carrier has not been designed yet.

BPE could also be used in other areas of surgery, in particular in maxillofacial surgery. Rupprecht et al. [98] treated the nonunion situation subsequent to primary mandible fracture by the application of BPE, compared with iliac crest bone grafts. The results demonstrated that BPE accelerated bone formation, especially in the initial stages of bone healing. Furthermore, antibiotic-containing BPE was suitable as a bone replacement material for the treatment of nonunion bone defects, suggesting that this joint action could be effective for osteomyelitis. Another BPE successfully applied in oral surgery was reported by Kuvat et al., which verified the effects of BFE not only by imaging but also by histological evaluation, which is not possible in humans generally [48]. Camargo [99] also obtained similar positive results. Although few studies have examined the use of BPE, it has been confirmed to have a strong capacity for bone formation, which is promising for its future application in clinical practice.

\section{Perspective for further application}

A biomaterial is a substance that has been engineered to take a form, which alone or as part of a complex system, can direct the course of any therapeutic or diagnostic procedure, in human or veterinary medicine, by controlling interactions with components of living systems [100]. BPE is an ideal biomaterial for bone formation because of the microenvironment it offers with its powerful osteogenic ability, which cannot be currently re-created by other means. Owing to this unique feature, BPE may be a very encouraging bone graft material for use in different areas. A few suggestions on the use of BPE are as follows:

The combination of BPE with other biomaterial or/and stem cells is the basis and precondition for its widespread application.

Spinal fusion: the good effect of BPE in ALIF has been observed in animal experiments and a few clinical reports; animal and pre-clinical experiments are necessary to determine its application in posteriolateral fusion.

Bone defects: designing a perfect carrier is imperative; the synergic effects of BPE and stem cells should be implemented during early, middle and later stages of bone formation in vivo; the combined effects of BPE and antibiotics (anti-tuberculosis medication in particular) on bone formation or stem cells should also be examined.

Artificial joint revision: whether BPE accelerates bone formation on the surface of different prostheses should be defined; the effects of BPE with impacted bone grafting around the joint should be measured in animal experiments.

\section{Conclusion}

BPE was regarded as the new generation of demineralized bone matrix because of its special and precise extracting process and unique biological characteristics. It has been verified to have bone formation capabilities by creating a microenvironment for osteogenesis. At present, BPE remains the most promising material for performing successful bone grafts.

This work was supported by the National Basic Research Program of China (Grant No. 2012CB619105), the National Natural Science Foundation of China (Grant Nos. 30571892, 81071512 and u0732001), and the Fabrikant Mads Clausens Foundation of Denmark.

1 Boyan B D, Ranly D M, McMillan J, et al. Osteoinductive ability of human allograft formulations. J Periodontol, 2006, 77: 1555-1563

2 Maus U, Andereya S, Gravius S, et al. How to store autologous bone graft perioperatively: an in vitro study. Arch Orthop Trauma Surg, 2008, 128: 1007-1011

3 Kruyt M C, van Gaalen S M, Oner F C, et al. Bone tissue engineering and spinal fusion: the potential of hybrid constructs by combining osteoprogenitor cells and scaffolds. Biomaterials, 2004, 25: 1463-1473

4 Wiltfang J, Kloss F R, Kessler P, et al. Effects of platelet-rich plasma on bone healing in combination with autogenous bone and bone substitutes in critical-size defects. An animal experiment. Clin Oral Implants Res, 2004, 15: 187-193

5 Heneghan H M, McCabe J P. Use of autologous bone graft in anterior cervical decompression: morbidity \& quality of life analysis. BMC Musculoskelet Disord, 2009, 10: 158

6 Catinella F P, De Laria G A, De Wald R L. False aneurysm of the 
superior gluteal artery. A complication of iliac crest bone grafting. Spine (Phila Pa 1976), 1990, 15: 1360-1362

7 Escalas F, DeWald R L. Combined traumatic arteriovenous fistula and ureteral injury: a complication of iliac bone-grafting. J Bone Joint Surg Am, 1977, 59: 270-271

8 Kurz L T, Garfin S R, Booth R J. Harvesting autogenous iliac bone grafts. A review of complications and techniques. Spine (Phila Pa 1976), 1989, 14: 1324-1331

9 Summers B N, Eisenstein S M. Donor site pain from the ilium. A complication of lumbar spine fusion. J Bone Joint Surg Br, 1989, 71: 677-680

10 Goulet J A, Senunas L E, DeSilva G L, et al. Autogenous iliac crest bone graft. Complications and functional assessment. Clin Orthop Relat Res, 1997, 339: 76-81

11 Fernyhough J C, Schimandle J J, Weigel M C, et al. Chronic donor site pain complicating bone graft harvesting from the posterior iliac crest for spinal fusion. Spine (Phila Pa 1976), 1992, 17: 1474-1480

12 Arribas-Garcia I, Alcala-Galiano A, Garcia A F, et al. Fracture of the anterior iliac crest following monocortical bone graft harvest in bisphosphonate-related mandibular pathological fracture: a case report. Oral Surg Oral Med Oral Pathol Oral Radiol Endod, 2009, 107: e12-14

13 Al-Sayyad M J, Abdulmajeed T M. Fracture of the anterior iliac crest following autogenous bone grafting. Saudi Med J, 2006, 27: 254-258

14 Cohn B T, Krackow K A. Fracture of the iliac crest following bone grafting. A case report. Orthopedics, 1988, 11: 473-474

15 Friend K D, Koval K J, Mirovsky Y, et al. Fracture of the iliac crest following bone grafting: a case report and literature review. Bull Hosp Jt Dis, 1995, 54: 49-51

16 Keller E E, Triplett W W. Iliac bone grafting: review of 160 consecutive cases. J Oral Maxillofac Surg, 1987, 45: 11-14

17 Zhukauskas R, Dodds R A, Hartill C, et al. Histological and radiographic evaluations of demineralized bone matrix and coralline hydroxyapatite in the rabbit tibia. J Biomater Appl, 2010, 24: 639-656

18 Katz J M, Nataraj C, Jaw R, et al. Demineralized bone matrix as an osteoinductive biomaterial and in vitro predictors of its biological potential. J Biomed Mater Res B Appl Biomater, 2009, 89: 127-134

19 Wang J C, Alanay A, Mark D, et al. A comparison of commercially available demineralized bone matrix for spinal fusion. Eur Spine J, 2007, 16: 1233-1240

20 Chen W J, Zhang F, Mustain W C, et al. Prefabrication of vascularized bone flap by demineralized bone matrix. J Craniofac Surg, 2007, 18: 43-48

21 Pietrzak W S, Woodell-May J, McDonald N. Assay of bone morphogenetic protein-2, -4 , and -7 in human demineralized bone matrix. J Craniofac Surg, 2006, 17: 84-90

22 Sundar S, Pendegrass C J, Blunn G W. Tendon bone healing can be enhanced by demineralized bone matrix: a functional and histological study. J Biomed Mater Res B Appl Biomater, 2009, 88: 115-122

23 Liu G, Li Y, Sun J, et al. In vitro and in vivo evaluation of osteogenesis of human umbilical cord blood-derived mesenchymal stem cells on partially demineralized bone matrix. Tissue Eng Part A, 2010, 16: 971-982

24 Price C T, Connolly J F, Carantzas A C, et al. Comparison of bone grafts for posterior spinal fusion in adolescent idiopathic scoliosis. Spine (Phila Pa 1976), 2003, 28: 793-798

25 Senn N. Senn on the healing of aseptic bone cavities by implantation of antiseptic decalcified bone. Ann Surg, 1889, 5: 352-368

26 Urist M R. Bone: formation by autoinduction. Science, 1965, 150: 893-899

27 Block J E, Poser J. Does xenogeneic demineralized bone matrix have clinical utility as a bone graft substitute? Med Hypotheses, 1995, 45: $27-32$

28 Jensen J, Foldager C B, Jakobsen T V, et al. Use of carboxymethyl cellulose and collagen carrier with equine bone lyophilisate suggests late onset bone regenerative effect in a humerus drill defect-a pilot study in six sheep. Open Orthop J, 2010, 4: 181-187

29 Nienhuijs M E, Walboomers X F, Briest A, et al. Healing of bone defects in the goat mandible, using COLLOSS $\mathrm{E}$ and beta-tricalciumphosphate. J Biomed Mater Res B Appl Biomater, 2010, 92: 517-524

30 Huffer W E, Benedict J J, Turner A S, et al. Repair of sheep long bone cortical defects filled with COLLOSS, COLLOSS E, OSSAPLAST, and fresh iliac crest autograft. J Biomed Mater Res B Appl Biomater, 2007, 82: 460-470

31 Termine J D, Belcourt A B, Conn K M, et al. Mineral and collagen-binding proteins of fetal calf bone. J Biol Chem, 1981, 256: 10403-10408

32 Nienhuijs M E, Poulsen K, van der Zande M, et al. Analytical assessment of the osteoinductive material COLLOSSE. J Biomed Mater Res B Appl Biomater, 2009, 89: 300-305

33 Delmas P D, Tracy R P, Riggs B L, et al. Identification of the noncollagenous proteins of bovine bone by two-dimensional gel electrophoresis. Calcif Tissue Int, 1984, 36: 308-316

34 Wildemann B, Kadow-Romacker A, Pruss A, et al. Quantification of growth factors in allogenic bone grafts extracted with three different methods. Cell Tissue Bank, 2007, 8: 107-114

35 Wildemann B, Kadow-Romacker A, Haas N P, et al. Quantification of various growth factors in different demineralized bone matrix preparations. J Biomed Mater Res A, 2007, 81: 437-442

36 Honsawek S, Powers R M, Wolfinbarger L. Extractable bone morphogenetic protein and correlation with induced new bone formation in an in vivo assay in the athymic mouse model. Cell Tissue Bank, 2005, 6: 13-23

37 Bae H W, Zhao L, Kanim L E, et al. Intervariability and intravariability of bone morphogenetic proteins in commercially available demineralized bone matrix products. Spine (Phila Pa 1976), 2006, 31: 1299-1306; discussion 1307-1308

38 Carrington J L, Roberts A B, Flanders K C, et al. Accumulation, localization, and compartmentation of transforming growth factor beta during endochondral bone development. J Cell Biol, 1988, 107: 1969-1975

39 Bachra B N. Calcification in vitro of demineralized bone matrix. Electron microscopic and chemical aspects. Calcif Tissue Res, 1972, 8: $287-303$

40 Hanson D A, Eyre D R. Molecular site specificity of pyridinoline and pyrrole cross-links in type I collagen of human bone. J Biol Chem, 1996, 271: 26508-26516

41 Deutsch H. High-dose bone morphogenetic protein-induced ectopic abdomen bone growth. Spine J, 2010, 10: e1-4

42 Boraiah S, Paul O, Hawkes D, et al. Complications of recombinant human BMP-2 for treating complex tibial plateau fractures: a preliminary report. Clin Orthop Relat Res, 2009, 467: 3257-3262

43 Brower R S, Vickroy N M. A case of psoas ossification from the use of BMP-2 for posterolateral fusion at L4-L5. Spine (Phila Pa 1976), 2008, 33: E653-655

44 Lee K J, Roper J G, Wang J C. Demineralized bone matrix and spinal arthrodesis. Spine J, 2005, 5: 217S-223S

$45 \mathrm{Li} \mathrm{H}$, Zou X, Woo C, et al. Experimental anterior lumbar interbody fusion with an osteoinductive bovine bone collagen extract. Spine (Phila Pa 1976), 2005, 30: 890-896

46 Walboomers X F, Jansen J A. Bone tissue induction, using a COLLOSS-filled titanium fibre mesh-scaffolding material. Biomaterials, 2005, 26: 4779-4785

47 Huffer W E, Benedict J J, Rettenmaier R, et al. Osteoinduction with COLLOSS, COLLOSS E, and GFm. Adv Exp Med Biol, 2006, 585: $87-100$

48 Kuvat S V, Cizmeci O, Bicer A, et al. Improving bony stability in maxillofacial surgery: use of osteogenetic materials in patients with profound $(>$ or $=5 \mathrm{~mm}$ ) maxillary advancement, a clinical study. $\mathrm{J}$ Plast Reconstr Aesthet Surg, 2009, 62: 639-645

49 Rodrigues C V, Serricella P, Linhares A B, et al. Characterization of a bovine collagen-hydroxyapatite composite scaffold for bone tissue engineering. Biomaterials, 2003, 24: 4987-4997

50 Grzesik W J, Robey P G. Bone matrix RGD glycoproteins: immunolocalization and interaction with human primary osteoblastic bone cells in vitro. J Bone Miner Res, 1994, 9: 487-496 
51 Anselme K. Osteoblast adhesion on biomaterials. Biomaterials, 2000, 21: 667-681

52 Becerra J, Andrades J A, Ertl D C, et al. Demineralized bone matrix mediates differentiation of bone marrow stromal cells in vitro: effect of age of cell donor. J Bone Miner Res, 1996, 11: 1703-1714

53 Vilalta M, Jorgensen C, Degano I R, et al. Dual luciferase labelling for non-invasive bioluminescence imaging of mesenchymal stromal cell chondrogenic differentiation in demineralized bone matrix scaffolds. Biomaterials, 2009, 30: 4986-4995

54 Liu G, Sun J, Li Y, et al. Evaluation of partially demineralized osteoporotic cancellous bone matrix combined with human bone marrow stromal cells for tissue engineering: an in vitro and in vivo study. Calcif Tissue Int, 2008, 83: 176-185

55 Gurevitch O, Kurkalli B G, Prigozhina T, et al. Reconstruction of cartilage, bone, and hematopoietic microenvironment with demineralized bone matrix and bone marrow cells. Stem Cells, 2003, 21: 588-597

56 Muschler G F, Matsukura Y, Nitto H, et al. Selective retention of bone marrow-derived cells to enhance spinal fusion. Clin Orthop Relat Res, 2005, 432: 242-251

57 Ouyang H W, Cao T, Zou X H, et al. Mesenchymal stem cell sheets revitalize nonviable dense grafts: implications for repair of large-bone and tendon defects. Transplantation, 2006, 82: 170-174

58 Tsiridis E, Ali Z, Bhalla A, et al. In vitro and in vivo optimization of impaction allografting by demineralization and addition of rh-OP-1. J Orthop Res, 2007, 25: 1425-1437

59 Liu G, Shu C, Cui L, et al. Tissue-engineered bone formation with cryopreserved human bone marrow mesenchymal stem cells. Cryobiology, 2008, 56: 209-215

60 Breitbart E A, Meade S, Azad V, et al. Mesenchymal stem cells accelerate bone allograft incorporation in the presence of diabetes mellitus. J Orthop Res, 2010, 28: 942-949

61 Woo C, Li H, Baatrup A, et al. Effects of bone protein extract on human mesenchymal stem cells proliferation and differentiation. J Biomed Mater Res A, 2006, 79: 552-556

62 Kasten P, Luginbuhl R, van Griensven M, et al. Comparison of human bone marrow stromal cells seeded on calcium-deficient hydroxyapatite, beta-tricalcium phosphate and demineralized bone matrix. Biomaterials, 2003, 24: 2593-2603

63 Krugliakov P V, Sokolova I B, Zin'Kova N N, et al. The influence of mesenchymal stem cells on bone tissue regeneration upon implantation of demineralized bone matrix. Tsitologiia, 2005, 47: 466-477

64 Kruglyakov P V,. Sokolova I B, Zin'Kova N N, et al. Effect of mesenchymal stem cells on rejection of xenogenic bone transplant. Bull Exp Biol Med, 2006, 142: 534-537

65 Honsawek S, Dhitiseith D, Phupong V. Effects of demineralized bone matrix on proliferation and osteogenic differentiation of mesenchymal stem cells from human umbilical cord. J Med Assoc Thai, 2006, 89: S189-195

66 El-Sabban M E, El-Khoury H, Hamdan-Khalil R, et al. Xenogenic bone matrix extracts induce osteoblastic differentiation of human bone marrow-derived mesenchymal stem cells. Regen Med, 2007, 2: 383-390

67 Torricelli P, Fini M, Giavaresi G, et al. In vitro osteoinduction of demineralized bone. Artif Cells Blood Substit Immobil Biotechnol, 1998, 26: 309-315

68 Peel S A, Hu Z M, Clokie C M. In search of the ideal bone morphogenetic protein delivery system: in vitro studies on demineralized bone matrix, purified, and recombinant bone morphogenetic protein. J Craniofac Surg, 2003, 14: 284-291

69 Li H, Springer M, Zou X, et al. Ectopic bone induction by equine bone protein extract. Adv Exp Med Biol, 2006, 585: 393-402

70 Joseph V, Rampersaud Y R. Heterotopic bone formation with the use of rhBMP2 in posterior minimal access interbody fusion: a CT analysis. Spine (Phila Pa 1976), 2007, 32: 2885-2890

71 Baas J, Elmengaard B, Bechtold J, et al. Ceramic bone graft substitute with equine bone protein extract is comparable to allograft in terms of implant fixation: a study in dogs. Acta Orthop, 2008, 79: $841-850$
72 Nienhuijs M E, Walboomers X F, Gelinsky M, et al. The evaluation of mineralized collagen as a carrier for the osteoinductive material COLLOSS $^{\circledR}$ E, in vivo. Tissue Eng Part A, 2011, 17: 1683-1690

73 Lode A, Bernhardt A, Kroonen K, et al. Development of a mechanically stable support for the osteoinductive biomaterial COLLOSS E. J Tissue Eng Regen Med, 2009, 3: 149-152

74 Zou X, Zou L, Foldager C, et al. Different mechanisms of spinal fusion using equine bone protein extract, rhBMP-2 and autograft during the process of anterior lumbar interbody fusion. Biomaterials, 2009, 30: 991-1004

75 Khan M T, Stockley I, Ibbotson C. Allograft bone transplantation: a Sheffield experience. Ann R Coll Surg Engl, 1998, 80: 150-153

76 Donati D, Colangeli M, Colangeli S, et al. Allograft-prosthetic composite in the proximal tibia after bone tumor resection. Clin Orthop Relat Res, 2008, 466: 459-465

77 Yoshida Y, Osaka S, Mankin H J. Hemipelvic allograft reconstruction after periacetabular bone tumor resection. J Orthop Sci, 2000, 5: 198-204

78 Li H, Zou X, Springer M, et al. Instrumented anterior lumbar interbody fusion with equine bone protein extract. Spine (Phila Pa 1976), 2007, 32: E126-129

79 Baas J, Lamberg A, Jensen T B, et al. The bovine bone protein lyophilisate Colloss improves fixation of allografted implants - an experimental study in dogs. Acta Orthop, 2006, 77: 791-798

80 Nienhuijs M E, Walboomers X F, Merkx M A, et al. Bone-like tissue formation using an equine COLLOSS E-filled titanium scaffolding material. Biomaterials, 2006, 27: 3109-3114

81 van der Zande M, Walboomers X F, Briest A, et al. The effect of combined application of TGFbeta-1, BMP-2, and COLLOSS E on the development of bone marrow derived osteoblast-like cells in vitro. J Biomed Mater Res A, 2008, 86: 788-795

82 Alanay A, Wang J C, Shamie A N, et al. A novel application of high-dose $(50 \mathrm{kGy})$ gamma irradiation for demineralized bone matrix: effects on fusion rate in a rat spinal fusion model. Spine J, 2008, 8: 789-795

83 Dodds R A, York-Ely A M, Zhukauskas R, et al. Biomechanical and radiographic comparison of demineralized bone matrix, and a coralline hydroxyapatite in a rabbit spinal fusion model. J Biomater Appl, 2010, 25: 195-1215

84 Urrutia J, Thumm N, Apablaza D, et al. Autograft versus allograft with or without demineralized bone matrix in posterolateral lumbar fusion in rabbits. Laboratory investigation. J Neurosurg Spine, 2008, 9: 84-89

85 Lee Y P, Jo M, Luna M, et al. The efficacy of different commercially available demineralized bone matrix substances in an athymic rat model. J Spinal Disord Tech, 2005, 18: 439-444

86 Qiu Q Q, Shih M S, Stock K, et al. Evaluation of DBM/AM composite as a graft substitute for posterolateral lumbar fusion. J Biomed Mater Res B Appl Biomater, 2007, 82: 239-245

87 Li H, Zou X, Laursen M, et al. The influence of intervertebral disc tissue on anterior spinal interbody fusion: an experimental study on pigs. Eur Spine J, 2002, 11: 476-481

$88 \mathrm{Li} \mathrm{H}$, Zou X, Xue Q, et al. Anterior lumbar interbody fusion with carbon fiber cage loaded with bioceramics and platelet-rich plasma. An experimental study on pigs. Eur Spine J, 2004, 13: 354-358

89 Li H, Zou X, Woo C, et al. Experimental lumbar spine fusion with novel tantalum-coated carbon fiber implant. J Biomed Mater Res B Appl Biomater, 2007, 81: 194-200

90 Foldager C, Bendtsen M, Zou X, et al. ISSLS prize winner: positron emission tomography and magnetic resonance imaging for monitoring interbody fusion with equine bone protein extract, recombinant human bone morphogenetic protein-2, and autograft. Spine (Phila $\mathrm{Pa}$ 1976), 2008, 33: 2683-2690

91 Foldager C, Bendtsen M, Nygaard J V, et al. Differences in early osteogenesis and bone micro-architecture in anterior lumbar interbody fusion with rhBMP-2, equine bone protein extract, and autograft. Bone, 2009, 45: 267-273

92 Kloss F R, Schlegel K A, Felszeghy E, et al. Applying an osteoin- 
ductive protein complex for regeneration of osseous defects. Mund Kiefer Gesichtschir, 2004, 8: 12-17

93 Schlegel K A, Kloss F R, Kessler P, et al. Bone conditioning to enhance implant osseointegration: an experimental study in pigs. Int $\mathrm{J}$ Oral Maxillofac Implants, 2003, 18: 505-511

94 Schlegel K A, Thorwarth M, Plesinac A, et al. Expression of bone matrix proteins during the osseus healing of topical conditioned implants: an experimental study. Clin Oral Implants Res, 2006, 17: 666-672

95 Baas J. Adjuvant therapies of bone graft around non-cemented experimental orthopedic implants stereological methods and experiments in dogs. Acta Orthop Suppl, 2008, 79: 1-43

96 Swenson C L, Arnoczky S P. Demineralization for inactivation of infectious retrovirus in systemically infected cortical bone: in vitro and in vivo experimental studies. J Bone Joint Surg Am, 2003, 85-A: 323-332

97 Bertagnoli R. Osteoinductive bone regeneration substance Colloss in spinal fusion. Eur Spine J, 2002, 11: 189-190

98 Rupprecht S, Petrovic L, Burchhardt B, et al. Antibiotic-containing collagen for the treatment of bone defects. J Biomed Mater Res B Appl Biomater, 2007, 83: 314-319

99 Camargo P M, Wolinsky L E, Burgess A V, et al. Bovine-derived bone protein extract in the treatment of mandibular Class II furcations. Compend Contin Educ Dent, 2002, 23: 1023-1028, 1030, passim 1032; quiz 1042

100 Williams D F. On the nature of biomaterials. Biomaterials, 2009, 30: 5897-5909

101 Baas J, Jakobsen T, Elmengaard B, et al. The effect of adding an equine bone matrix protein lyophilisate on fixation and osseointegration of HA-coated Ti implants. J Biomed Mater Res A, 2012, 100: 188-194

102 Ding M, Rojskjaer J, Cheng L, et al. The effects of a novel-reinforced bone substitute and Colloss ${ }^{\circledR} \mathrm{E}$ on bone defect healing in sheep. J Biomed Mater Res B Appl Biomater, 2012, 100B: 1826-1835

Open Access This article is distributed under the terms of the Creative Commons Attribution License which permits any use, distribution, and reproduction in any medium, provided the original author(s) and source are credited. 